\title{
Article \\ C-Reactive Protein to Albumin Ratio as Prognostic Marker in Locally Advanced Non-Small Cell Lung Cancer Treated with Chemoradiotherapy
}

\author{
Alina Frey ${ }^{1}$, Daniel Martin ${ }^{1,2,3,4}$, Louisa D'Cruz ${ }^{1}$, Emmanouil Fokas ${ }^{1,2,3,4}$, Claus Rödel ${ }^{1,2,3,4}$ \\ and Maximilian Fleischmann ${ }^{1, *}$ \\ 1 Department of Radiation Oncology, Hospital of the Johann Wolfgang Goethe University, \\ 60590 Frankfurt, Germany; alina.frey@kgu.de (A.F.); daniel.martin@kgu.de (D.M.); dcruzla@gmail.com (L.D.); \\ emmanouil.fokas@kgu.de (E.F.); clausmichael.roedel@kgu.de (C.R.) \\ 2 German Cancer Research Center (DKFZ), 69120 Heidelberg, Germany \\ 3 German Cancer Consortium (DKTK), Partner Site Frankfurt am Main, 60590 Frankfurt, Germany \\ 4 Frankfurt Cancer Institute, 60590 Frankfurt, Germany \\ * Correspondence: maximilian.fleischmann@kgu.de
}

\section{check for} updates

Citation: Frey, A.; Martin, D.; D'Cruz, L.; Fokas, E.; Rödel, C.; Fleischmann, M. C-Reactive Protein to Albumin Ratio as Prognostic Marker in Locally Advanced Non-Small Cell Lung Cancer Treated with Chemoradiotherapy. Biomedicines 2022, 10, 598. https://doi.org/ 10.3390/biomedicines10030598 Academic Editors: Chih-Jen Yang and Hsiang-Hao Chuang

Received: 14 February 2022 Accepted: 28 February 2022 Published: 3 March 2022

Publisher's Note: MDPI stays neutral with regard to jurisdictional claims in published maps and institutional affiliations.

Copyright: (C) 2022 by the authors. Licensee MDPI, Basel, Switzerland. This article is an open access article distributed under the terms and conditions of the Creative Commons Attribution (CC BY) license (https:// creativecommons.org/licenses/by/ $4.0 /)$.

\begin{abstract}
Despite the implementation of consolidative immune checkpoint inhibition after definitive chemoradiotherapy (CRT), the prognosis for locally advanced non-small-cell lung cancer (NSCLC) remains poor. We assessed the impact of the C-reactive protein (CRP) to albumin ratio (CAR) as an inflammation-based prognostic score in patients with locally advanced NSCLC treated with CRT. We retrospectively identified and analyzed 52 patients with primary unresectable NSCLC (UICC Stage III) treated with definitive/neoadjuvant CRT between 2014 and 2019. CAR was calculated by dividing baseline CRP by baseline albumin levels and correlated with clinicopathologic parameters to evaluate prognostic impact. After dichotomizing patients by the median, univariate and multivariate Cox regression analyses were performed. An increased CAR was associated with advanced T-stage $(p=0.018)$ and poor performance status $(p=0.004)$. Patients with pre-therapeutic elevated CAR had significantly lower hemoglobin and higher leukocyte levels (hemoglobin $p=0.001$, leukocytes $p=0.018$ ). High baseline CAR was shown to be associated with worse local control (LPFS, $p=0.006$ ), shorter progression-free survival (PFS, $p=0.038$ ) and overall survival (OS, $p=0.022$ ), but not distant metastasis-free survival (DMFS). Multivariate analysis confirmed an impaired outcome in patients with high CAR (LPFS: HR 3.562, 95\% CI 1.294-9.802, $p=0.011$ ). CAR is an easily available and independent prognostic marker after CRT in locally advanced NSCLC. CAR may be a useful biomarker for patient stratification to individualize treatment concepts.
\end{abstract}

Keywords: NSCLC; chemoradiotherapy; CRT; CAR; C-reactive protein; albumin; inflammation; prognostic; predictive; biomarker

\section{Introduction}

Non-small-cell lung cancer (NSCLC) impacts more than 2.2 million people annually, representing the most common cause of cancer-related death worldwide [1]. At the time of diagnosis, approximately one-third of patients are diagnosed with Union for International Cancer Control (UICC) stage III disease [2]. Concurrent platinum-based doublet chemoradiotherapy (CRT), followed by immune checkpoint inhibition (ICI) for patients without disease progression after CRT, remains the standard of care for patients with primary unresectable locally advanced NSCLC. Updated survival analyses from the placebo-controlled phase III PACIFIC trial presented at the 2021 ASCO Annual Meeting have shown that sequential ICI with durvalumab resulted in a durable benefit for progression-free (PFS) and overall survival (OS) at 5 years [3]. However, in this cohort, OS rates were $42.9 \%$ and $33.4 \%$ (stratified HR $0.72,95 \%$ CI $0.59-0.89$; median 47.5 vs. 29.1 months), and PFS rates 
were $33.1 \%$ and $19.0 \%$ (stratified HR $0.55,95 \%$ CI $0.45-0.68$; median 16.9 vs. 5.6 months), respectively [3], indicating that further efforts are necessary to improve outcomes and survival. In this context, accurate risk stratification based on robust biomarkers could be a strategy to further improve and individualize treatment concepts.

The interplay between host immune response and cancer progression has been studied intensively for decades [4-6]. Cancer triggers systemic inflammation with a corresponding increase in pro-inflammatory cytokines such as interleukin 6 (IL6) and tumor necrosis factor $\alpha(\mathrm{TNF} \alpha)$ [4-6]. Upon cytokine stimulation, acute phase proteins including the C-reactive protein (CRP) are synthesized in hepatocytes [7]. CRP activates the complement system, initiates humoral and cellular effector mechanisms of the innate immune system, and is a common and routinely measured marker of inflammation [8].

Inflammatory cytokines lead to increased capillary permeability [9] and subsequently to a loss of serum albumin into the interstitium [10], while altered protein synthesis capacity contributes to a decrease in albumin synthesis during inflammation [11]. Therefore, CRP, and thus immune activation, correlates reciprocally with serum albumin levels [12,13], which is generally considered a marker of malnutrition and liver function [10].

An increased CRP to albumin ratio (CAR) has already been reported to be significantly associated with worse survival in various malignancies, including head and neck cancers and colorectal, anal and bladder cancer [14-17]. Previous studies on CAR in NSCLC have focused on early-stage or primary resectable disease, palliative regimens and second-line treatment [18-20]. More recently, Yang et al. further strengthened the negative prognostic impact of elevated baseline CAR in a prospective analysis of a heterogeneous cohort of 387 NSCLC patients [21].

Similar to CAR, the Glasgow Prognostic Score (GPS) reflects on CRP and albumin levels and has been explored in various settings. In brief, the results were consistent with those of CAR, and elevated GPS was associated with poorer survival in patients with NSCLC [22-24].

We here investigated the correlation of pretherapeutic CAR as a biomarker for inflammation and malnutrition with clinicopathologic features and its prognostic value in patients with locally advanced and primary unresectable UICC stage III NSCLC treated with CRT. In addition, to account for GPS and to compare its prognostic value with that of CAR, we performed corresponding survival analyses.

\section{Materials and Methods}

\subsection{Patients and Treatment Protocol}

We retrospectively identified 52 patients with locally advanced and primary unresectable NSCLC (UICC III) treated between 1/2014 and 12/2019 with definitive or neoadjuvant-intended CRT. Pseudonymized data were used after institutional ethics committee approval.

Staging was routinely performed, including clinical examination and pulmonary function testing, computed tomography (CT) of the chest and abdomen and positron emission tomography/computed tomography (PET/CT). For brain metastases, screening magnetic resonance imaging (MRI) was performed. Histological confirmation and pathological classification were provided after bronchoscopic, thoracoscopic or CT-guided biopsy. The decision on definitive versus neoadjuvant-intended CRT and potential operability was made by a multidisciplinary tumor board, with consideration of the patient's preferences.

Radiotherapy (RT) was applied using either 3D-conformal radiotherapy or intensitymodulated RT (IMRT). Patients were treated with a median total dose of 66.6 Gray (Gy) (range: 45-66.6 Gy) with daily fractions of 1.8 or 2 Gy five days per week. Patients primarily assigned to neoadjuvant-intended CRT were treated with a median dose of 50.4 Gy (range: 45-59.4 Gy). Except for one patient, all patients received RT as planned without dose reduction.

Concurrent chemotherapy consisted of platinum-based chemotherapy and vinorelbine. Platinum-based chemotherapy was administered in the first and fifth week of RT. Generally, 
cisplatin (20 mg/m², day 1-5 and day 28-33) was preferred. For patients not eligible for cisplatin-based chemotherapy for various reasons (e.g., renal insufficiency), carboplatin (AUC1, day 1-5 and day 28-33) was applied. Vinorelbine (50 $\mathrm{mg} / \mathrm{m}^{2}$, per os) was given on days $1,8,15,29,36$ and 43 . Etoposide $\left(90 \mathrm{mg} / \mathrm{m}^{2}\right.$, day $1-3$ and day 28-31) was used instead of vinorelbine, e.g., in patients with neuroendocrine differentiation. Seventeen patients received induction chemotherapy before CRT. Induction chemotherapy had no effect on OS $(p=0.837)$; thus, these patients were included. In addition, no differences in OS were found between patients who received neoadjuvant-intended or definitive CRT ( $p=0.758)$, so these patients were also included. Eight of nine patients who received neoadjuvant-intended treatment underwent surgery.

\subsection{Response Assessment and Follow-Up}

Initial response assessment and restaging procedures including pulmonary function testing and imaging (CT scans of chest and abdomen) were performed 6-8 weeks after completion of CRT. In the first year after the completion of therapy, follow-up examinations were scheduled every three months, afterwards every six or twelve months or inter-individually depending on the respective risk profile and clinical history.

\subsection{Clinicopathologic Features and Serum Chemistry}

Personal data and clinicopathologic features were retrieved from the patient files of our hospital database including sex and age, general physical condition at the time of diagnosis according to the Eastern Cooperative Oncology Group (ECOG) Performance Status, TNM stage, UICC stage and histology. Height and weight were documented at the beginning of the therapy; body mass index (BMI) was calculated by dividing weight in kilograms by height in meters squared $\left(\mathrm{kg} / \mathrm{m}^{2}\right)$. Blood parameters were obtained as part of routine diagnostics at the start of therapy. The baseline was either defined on the day of therapy initiation or up to 9 days before treatment.

CAR was calculated by dividing CRP in $\mathrm{mg} / \mathrm{dL}$ through albumin in $\mathrm{g} / \mathrm{dL}$. GPS was determined according to Forrest et al. [25]. Patients with CRP values $\leq 1.0 \mathrm{mg} / \mathrm{dL}$ and albumin values $\geq 3.5 \mathrm{~g} / \mathrm{dL}$ were categorized as GPS 0 , CRP levels $>1.0 \mathrm{mg} / \mathrm{dL}$ or albumin levels $<3.5 \mathrm{~g} / \mathrm{dL}$ as GPS 1, and CRP levels $>1.0 \mathrm{mg} / \mathrm{dL}$ and albumin levels $<3.5 \mathrm{~g} / \mathrm{dL}$ as GPS 2. Leukocyte and platelet counts per nanoliter (nL), as well as hemoglobin in $\mathrm{g} / \mathrm{dL}$, were recorded regularly to define the patient-specific nadir (defined as lowest blood count from start to 4 weeks after completion of CRT).

\subsection{Statistical Analysis}

Differences between groups regarding continuous variables were tested for statistical significance via the Mann-Whitney $U$ test for independent samples. Survival times were calculated from the start of CRT to the date of respective events or last follow-up. Events were defined as death from any cause (overall survival, OS), and progression was defined as local recurrence/progression after remission/stable disease, or distant metastasis (progression-free survival, PFS), isolated local recurrence/progression (local progressionfree survival, LPFS), or distant metastasis (distant metastasis-free survival, DMFS). Survival time analyses were plotted according to the Kaplan-Meier method and the log-rank test was used for calculation.

We further performed multivariate Cox analyses with the calculation of hazard ratios (HRs) and corresponding 95\% confidence intervals (CIs) to investigate the influence of high or low CAR (cut-off: median) and other categorical predictor variables (cut-off for not already dichotomous variables: median, standard values or laboratory limits) on survival. For reliable assessment of the results of multivariate Cox analysis, at least ten events should occur for each prognostic variable included [26]. Due to the low number of events, we only included prognostic variables in the multivariate model that were statistically significant in the univariate Cox analysis. All statistical analysis was performed using IBM SPSS Version 
27 (Armonk, NY, USA). All tests were two-sided, and a $p$-value of 0.05 was considered statistically significant.

\section{Results}

\subsection{Patient Characteristics}

We retrospectively assessed 52 patients (female $n=27,51.9 \%$ ) with locally advanced and primary unresectable UICC stage III NSCLC (T1-4, N0-3, M0). Median age was 66 (range: 47-79) years. Median follow-up was 17 (range: 2-76) months. Twenty-eight of fifty-two patients $(53.8 \%)$ had a CAR $\leq$ the median. Detailed pre-treatment patient characteristics are summarized in Table 1.

Table 1. Patient, disease, treatment, blood characteristics.

\begin{tabular}{|c|c|c|c|}
\hline & & & Median or $n$ (Range or \%) \\
\hline \multirow[t]{7}{*}{ Patients } & Sex & Male & $25(48.1)$ \\
\hline & & Female & $27(51.9)$ \\
\hline & Age (years) & & $66(47-79)$ \\
\hline & $\operatorname{BMI}\left(\mathrm{kg} / \mathrm{m}^{2}\right)$ & & $25.14(16.48-39.12)$ \\
\hline & ECOG & 0 & $16(30.8)$ \\
\hline & & 1 & $30(57.7)$ \\
\hline & & 2 & $6(11.5)$ \\
\hline \multirow[t]{13}{*}{ Tumor } & T-stage & $\mathrm{T} 1$ & $4(7.7)$ \\
\hline & & $\mathrm{T} 2$ & $14(26.9)$ \\
\hline & & $\mathrm{T} 3$ & $16(30.8)$ \\
\hline & & $\mathrm{T} 4$ & $18(34.6)$ \\
\hline & N-stage & No & $6(11.5)$ \\
\hline & & N1 & $2(3.9)$ \\
\hline & & N2 & $26(50.0)$ \\
\hline & & N3 & $18(34.6)$ \\
\hline & Histology & Adeno & $29(55.8)$ \\
\hline & & Squamous cell & $17(32.7)$ \\
\hline & & Spindle cell (sarcomatoid) & $1(1.9)$ \\
\hline & & Neuroendocrine (large cell) & $3(5.8)$ \\
\hline & & NOS & $2(3.8)$ \\
\hline \multirow[t]{5}{*}{ Treatment } & CRT & Definitive & $43(82.7)$ \\
\hline & & Neoadjuvant-intended & 9 (17.3) \\
\hline & Induction $\mathrm{CT}$ & Yes & $17(32.7)$ \\
\hline & & No & $35(67.3)$ \\
\hline & Total dose (Gy) & & $66.6(45-66.6)$ \\
\hline \multirow[t]{9}{*}{ Baseline } & $\mathrm{CRP}(\mathrm{mg} / \mathrm{dL})$ & & $1.37(0.03-21.43)$ \\
\hline & Albumin (g/dL) & & $4.1(2.8-4.9)$ \\
\hline & CAR & & $0.32(0.01-7.14)$ \\
\hline & Hemoglobin (g/dL) & & $12.35(8.0-15.2)$ \\
\hline & Leukocytes $(/ \mathrm{nL})$ & & 8.39 (3.29-80.95) \\
\hline & Thrombocytes (/nL) & & 312.5 (117-679) \\
\hline & GPS & 0 & $20(38.5)$ \\
\hline & & 1 & $29(55.8)$ \\
\hline & & 2 & $3(5.8)$ \\
\hline \multirow[t]{3}{*}{ Nadir } & Hemoglobin (g/dL) & & $10.0(6.0-12.3)$ \\
\hline & Leukocytes $(/ \mathrm{nL})$ & & $2.28(0.15-7.13)$ \\
\hline & Thrombocytes (/nL) & & $144(10-305)$ \\
\hline
\end{tabular}

Abbreviations: BMI, body mass index; ECOG, Eastern Cooperative Oncology Group Performance Status; NOS, not otherwise specified; CRT, chemoradiotherapy; CT, chemotherapy; Gy, Gray; CRP, C-reactive protein; CAR, CRP to albumin ratio; GPS, Glasgow Prognostic Score.

Higher T-stage (T1/2 vs. T3/4, $p=0.018)$ and poorer performance status ( $\geq$ ECOG 1 , $p=0.004$ ) were significantly associated with elevated baseline/pre-treatment CAR, whereas N-status (N0/1 vs. N2/3), sex, BMI (cut-off: $25 \mathrm{~kg} / \mathrm{m}^{2}$ ) and histology (adeno and squamous cell) were not $(p=0.265, p=0.763, p=0.971$ and $p=0.155)$ (Figure 1A). 

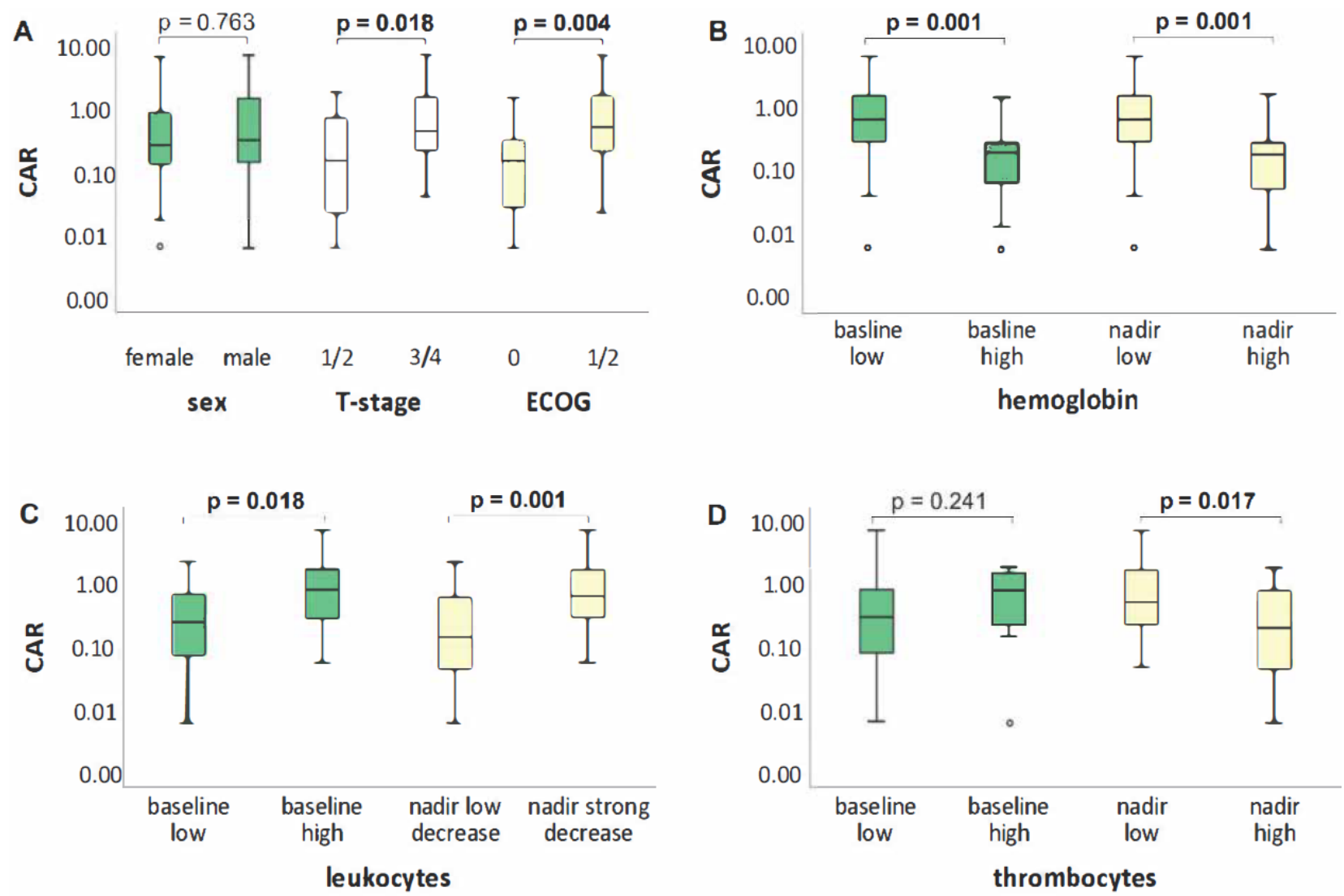

Figure 1. Differences in CAR by sex, T-stage, ECOG (A) and blood count (hemoglobin (B). leukocytes (C) and thrombocytes (D).

We examined associations between CAR as a continuous variable with baseline blood count and blood count at the patient-specific nadir. Significantly higher leukocyte counts (cut-off: $10 / \mathrm{nL}, p=0.018$ ) and lower hemoglobin levels (sex-specific cut-off for men: $13 \mathrm{~g} / \mathrm{dL}$, cut-off for women: $12 \mathrm{~g} / \mathrm{dL}, p=0.001$ ) were found at baseline in patients with a higher CAR. Platelet count at baseline showed no significant association with CAR (cutoff: $400 / \mathrm{nL}, p=0.241)$. In the patient-specific nadir, a more profound treatment-related leukocyte drop (cut-off: median, 6.2/nL, $p=0.001$ ), lower hemoglobin (cut-off: median, $10 \mathrm{~g} / \mathrm{dL}, p=0.001$ ) and thrombocyte values (cut-off: median, $144 / \mathrm{nL}, p=0.017$ ) were associated with a higher CAR (Figure 1B-D).

A higher baseline CAR was significantly associated with an advanced T-stage, poorer performance status (A), lower baseline hemoglobin values and higher baseline leukocyte counts. In the patient-specific nadir, elevated CAR was correlated to lower hemoglobin and thrombocyte levels and a stronger decrease in the leukocyte count.

\subsection{Clinical Outcomes: Disease Control and Survival}

\subsubsection{Univariate Analysis}

The OS and PFS at 3 years were $33 \%$ and $18 \%$, respectively. We have dichotomized the cohort at the median CAR of 0.32 to perform the survival analyses. Elevated baseline CAR ( $>$ median) was significantly associated with worse OS $(p=0.022)$, PFS $(p=0.038)$ and LPFS $(p=0.006)$, but not with DMFS $(p=0.207)$ (Figure 2$)$. The results of the Cox analysis are given in Table 2. In the univariate Cox analysis, only lower leukocyte levels in the patient-specific nadir (cut off: median, $2.3 / \mathrm{nL}, \mathrm{HR} 0.257,95 \% \mathrm{CI} 0.084-0.793, p=0.011$ ) and male sex (HR 2.711, 95\% CI 0.979-7.508, $p=0.046$ ) were significantly associated with worse LPFS (Supplementary Tables S1 and S2). 

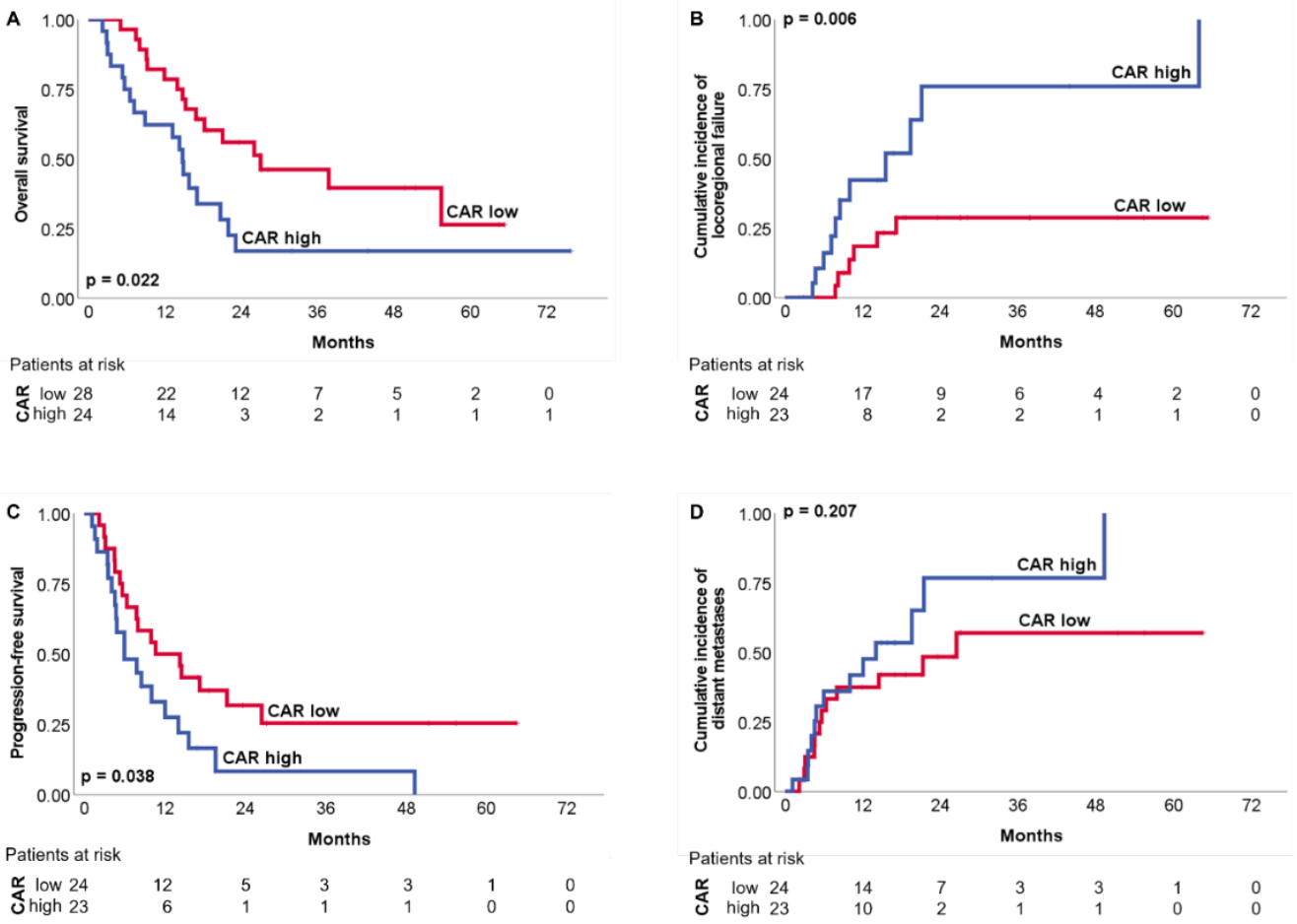

Figure 2. OS (A), LPFS (B), PFS (C) and DMFS (D) according to dichotomized CAR.

Table 2. Univariate Cox regression analysis with dichotomized CAR.

\begin{tabular}{lccc}
\hline & HR & $\mathbf{9 5 \%}$ CI & $p$-Value \\
\hline OS & 2.178 & $1.101-4.310$ & $\mathbf{0 . 0 2 2}$ \\
PFS & 2.005 & $1.026-3.918$ & $\mathbf{0 . 0 3 8}$ \\
LPFS & 3.723 & $1.365-10.151$ & $\mathbf{0 . 0 0 6}$ \\
DMFS & 1.658 & $0.749-3.671$ & 0.208 \\
\hline
\end{tabular}

Abbreviations: OS, overall survival; PFS, progression-free survival; LPFS, local progression-free survival; DMFS, distant metastasis-free survival.

Elevated baseline CAR was significantly associated with worse OS (A), LPFS (B) and PFS (C), but not DMFS (D). The number of patients at risk is given below.

In a next step, we created a subgroup excluding non-adenocarcinomas and nonsquamous cell carcinomas to further homogenize the patient cohort and to consider potential confounding factors. In this cohort, a high pre-therapeutic CAR was confirmed as significantly associated with an impaired OS (HR 2.268, 95\% CI 1.086-4.736, $p=0.025$ ), PFS (HR 2.105, 95\% CI 1.031-4.295, $p=0.037$ ) and LPFS (HR 4.426, 95\% CI 1.493-13.121, $p=0.004)$. Furthermore, in this subgroup, patients with squamous cell carcinoma (HR 2.456, 95\% CI 1.178-5.122, $p=0.013$ ) and a high BMI (cut-off: $25 \mathrm{~kg} / \mathrm{m}^{2}$, HR $2.161,95 \%$ CI 1.014-4.604, $p=0.041$ ) had a shorter OS. Poorer locoregional control was found in men (HR 3.303, 95\% CI 1.097-9.943, $p=0.025$ ), squamous cell carcinomas (HR 3.826, 95\% CI $1.304-11.225, p=0.009$ ) and low nadir leukocyte levels (HR 0.200, 95\% CI 0.056-0.712, $p=0.006)$. Univariate Cox regression analyses with corresponding outcome variables are given in Supplementary Table S1. Interestingly, GPS was not significantly associated with OS, PFS, LPFS or DMFS ( $p=0.441, p=0.634, p=0.222, p=0.800)$ (Supplementary Table S3).

\subsubsection{Multivariate Analysis}

Multivariate Cox regression analyses were only performed for LPFS and OS, as only outcome parameters that could be predicted by other variables in addition to CAR were listed. For PFS, CAR was the only significant predictor variable and DMFS could not be predicted by CAR in either subgroup. Stepwise backward elimination was chosen as the model for the multivariate analyses. Consequently, all independent variables that 
significantly predicted the outcome in univariate Cox regression analysis (Supplementary Table S2) were first included in the model and then successively removed if they did not contribute significantly. Values above $p>0.1$ were set as exclusion criteria for the variables in the model.

In the multivariate analyses, only CAR remained predictive for worse OS, whereas CAR and nadir leukocyte count remained predictive for adverse LPFS (CAR, cut-off: 0.32, median, HR 3.562, 95\% CI 1.294-9.802, $p=0.011$; nadir leukocyte count, cut-off: $2.3 / \mathrm{nl}$, median, HR $0.266,95 \%$ CI $0.085-0.836, p=0.013$ ) (Table 3).

Table 3. Multivariate Cox regression analysis.

\begin{tabular}{llccc}
\hline & & HR & $\mathbf{9 5 \%}$ CI & $p$-Value \\
\hline \multirow{2}{*}{ LPFS } & CAR & 3.562 & $1.294-9.802$ & $\mathbf{0 . 0 1 1}$ \\
& Leukocytes nadir & 0.266 & $0.085-0.836$ & $\mathbf{0 . 0 1 3}$ \\
& Sex & removed & & \\
\hline
\end{tabular}

OS was predicted by CAR only. Abbreviations: LPFS, local progression-free survival; CAR, C-reactive protein to albumin ratio.

Excluding non-adenocarcinomas and non-squamous cell carcinomas revealed a significant correlation of an elevated baseline CAR with a worse OS (HR 2.480, 95\% CI 1.178-5.220, $p=0.018$ ). BMI (cut-off: $25 \mathrm{~kg} / \mathrm{m}^{2}$, HR 2.359, 95\% CI 1.103-5.045, $p=0.024$ ) remained statistically significant (Supplementary Table S4).

\section{Discussion}

All stages of tumorigenesis are closely intertwined with inflammation [4]. Chronic inflammation contributes to tumor development by influencing the extracellular matrix, the tumor microenvironment and neoangiogenesis, thus promoting tumor growth and metastatic behavior [27]. CRP secreted by the liver upon IL6 stimulation is a dynamic laboratory parameter easily accessible for detecting and monitoring acute and chronic systemic inflammation as well as tumorigenic inflammatory response. It has been reported to be associated with impaired outcomes in oral and oropharyngeal cancer; gastrointestinal malignancies, including colorectal, pancreatic and hepatocellular cancer; as well as urological malignancies [28-32]. The prognostic impact of elevated CRP levels in patients with NSCLC has been investigated in various settings and several studies. In their meta-analysis of 1649 patients, Jin et al. analyzed eight studies and showed an association between elevated CRP levels and worse survival in patients with both primary resectable NSCLC and primary unresectable NSCLC [33].

Several more specific inflammatory scores based on cellular components of the blood have been explored for predicting response and survival in NSCLC [34,35]. For example, a high post-CRT neutrophil-to-lymphocyte ratio (NLR) was shown to be associated with significantly worse LPFS and OS in patients with locally-advanced NSCLC treated with definitive CRT $[36,37]$. Neutrophils can be stimulated by the tumor itself and-depending on their phenotypic and functional polarization-are considered tumor-promoting [38]. The neutrophil-to-lymphocyte ratio may represent the balance between pro-tumoral inflammatory status and anti-tumoral immune response. In addition to NLR, a lower lymphocyteto-monocyte ratio (LMR) has also been associated with an impaired prognosis in NSCLC patients [39]. Interestingly, an increasing LMR after nivolumab was related to an improved PFS in a cohort of 75 NSCLC patients, indicating LMR as a dynamic surrogate marker for response [40]. However, lymphocytopenia is a common side effect of radiation [41]. Therefore, a prognostic score that depends on the lymphocyte count could be considerably biased in patients who have undergone radiotherapy.

In contrast, serum albumin levels are a parameter of malnutrition and decrease during a systemic state of inflammation through increased CRP synthesis. The prevalence of malnutrition, cachexia and wasting, characterized by a significant weight loss, sarcopenia and decline in skeletal muscles in cancer patients, ranges from 30 to $80 \%$ and increases in terminal stages [42]. These facts indicate a strong interplay between nutrition, sarcopenia, 
inflammation and cancer progression. Thus, low albumin levels and nutrient indices have been shown to be associated with poorer outcomes in survival time analyses in various malignancies [43-45]. In two smaller cohorts, hypoalbuminemia has been shown to be a negative and independent prognostic factor in patients with metastatic or nonmetastatic NSCLC initially treated with chemotherapy or targeted therapies $[45,46]$. In addition, Rim et al. studied 353 elderly cancer patients treated with definitive RT and showed that low albumin levels were associated with a higher rate of poor treatment compliance [47]. In general, the state of systemic inflammation and worse nutritional status is often associated with higher tumor stages [48] and inferior survival in cancer patients [28-33,43-45], suggesting the potential of combined scores.

In the present study, we have assessed CAR as a prognostic and easily accessible biomarker in a homogenous cohort of 52 UICC stage III NSCLC patients treated uniformly with CRT. First, we have demonstrated that higher baseline CAR was significantly associated with the higher T stage (T1/2 vs. T3/4) and poor performance status (ECOG 0 vs. ECOG 1/2). Second, baseline CAR was significantly correlated to pre-treatment blood counts and the patient-specific nadir during CRT. Ultimately, elevated baseline CAR was associated with an impaired LPFS, PFS and OS. In multivariate analysis, CAR was shown to be an independent predictor of LPFS in the entire cohort. After exclusion of all non-squamous cell carcinomas and non-adenocarcinomas, CAR remained significant for OS. These results are consistent with previous studies and strengthen the prognostic impact of CAR [18-21].

As GPS was also reported as a prognostic marker for survival, we performed corresponding analyses for this score. However, GPS did not predict survival in our cohort. In contrast, Yotsukura et al. explored the prognostic impact of GPS in a large cohort of 1048 patients with primary resectable NSCLC. A high GPS was significantly associated with poor OS [22]. Kishi et al. reported GPS as a significant prognostic factor in a cohort of 165 patients with early-stage NSCLC treated with stereotactic body radiation therapy (SBRT) [23]. In a heterogeneously treated cohort of 261 unresectable NSCLC patients, high GPS was associated with poorer ECOG performance status and increased baseline leukocyte count. Moreover, GPS was associated with worse cancer-specific survival (CSS) [24]. A meta-analysis by Zhu et al., including 2669 patients from 12 studies, confirmed GPS as a predictor of survival in patients with NSCLC [49]. Interestingly, another meta-analysis by Jin et al., which evaluated the prognostic impact of GPS in 5817 patients from 11 studies, failed to show an association between GPS and worse survival among patients who underwent surgery [50]. In our cohort, we found albumin levels $<3.5 \mathrm{~g} / \mathrm{dL}$ in three patients only. Consistent with the findings of Matsumoto et al. [51], CAR appears to be a more reliable prognostic marker than GPS in cohorts where few patients have notably low albumin levels.

Unlike previous studies in which a higher BMI was associated with better survival rates [52], high BMI was associated with an inferior outcome in this study. One possible explanation is that patients with a high BMI in our cohort, e.g., in the context of metabolic syndrome, have known or initially undiagnosed preexisting conditions that have an unfavorable impact on the outcome. In addition, BMI does not necessarily reflect body composition and sarcopenia, which may have a greater impact on outcome and survival. However, in our cohort, only two patients were underweight $\left(B M I<18.5 \mathrm{~kg} / \mathrm{m}^{2}\right)$ and five were obese (BMI $\geq 30 \mathrm{~kg} / \mathrm{m}^{2}$ ), so caution should be used when interpreting these results. Adenocarcinomas were associated with prolonged OS, consistent with previous studies [53]. Furthermore, women had a better outcome, which is in line with other studies [1,54]. Low leukocyte levels during therapy, which may indicate increased toxicity [55], were associated with shorter survival in our cohort. As a result, a reduction in the chemotherapy dose may have been indicated, affecting disease control and survival.

Nevertheless, there are several limitations in the present study. Due to the retrospective design and the small sample size, confounding and sampling bias cannot be excluded. The results of multivariate Cox regression analysis require caution because of the relatively small number of events that occurred. We chose the median as the cut-off of the CAR. 
Previous studies using other statistical methods (e.g., receiver operating characteristic (ROC) curves) to determine cut-off values for CAR arrived at cut-off values from 0.0271 to 0.83 [14-21]. Further prospective studies could clarify which cut-off is most appropriate for CAR.

In summary, CAR is an easily accessible biomarker that reflects systemic inflammation and nutritional status and was associated with advanced tumor stage (T-stage), poor performance status and poor survival in patients with UICC stage III NSCLC. These findings may indicate that CAR is a tool for risk stratification and highlight the importance of early integration of interdisciplinary supportive care to improve treatment compliance and prognosis.

Supplementary Materials: The following supporting information can be downloaded at: https: //www.mdpi.com/article/10.3390/biomedicines10030598/s1, Table S1: Univariate Cox regression analysis; Table S2: Univariate Cox regression analysis for all variables (total cohort); Table S3: Univariate Cox regression analysis with GPS; Table S4: Multivariate Cox regression analysis for adenocarcinomas or squamous cell carcinomas only.

Author Contributions: M.F. and D.M. contributed to the conception and design of the study. A.F. and L.D. collected and organized data. A.F. performed the statistical analyses, designed the figures and wrote the first draft of the manuscript. M.F., D.M., E.F. and C.R. finalized the manuscript. All authors have read and agreed to the published version of the manuscript.

Funding: This project received no external funding.

Institutional Review Board Statement: The study was conducted according to the guidelines of the Declaration of Helsinki and approved by the Ethics Committee of the University Hospital Frankfurt, Germany (UCT-40-2020; 274/18).

Informed Consent Statement: Written informed consent for participation was not required for this study in accordance with the national legislation and the institutional requirements.

Data Availability Statement: Data are available upon reasonable request.

Conflicts of Interest: The authors declare no conflict of interest.

\section{Abbreviations}

CRT (chemoradiotherapy), NSCLC (non-small cell lung cancer), CRP (c-reactive protein), CAR (CRP to albumin ratio), UICC (Union for International Cancer Control), LPFS (local progression-free survival), PFS (progression-free survival), DMFS (distant metastasis-free survival), OS (overall survival), HR (hazard ratio), CI (confidence interval), ICI (immune checkpoint inhibition), IL6 (interleukin 6), TNF $\alpha$ (tumor necrosis factor $\alpha$ ), GPS (Glasgow Prognostic Score), CT (computed tomography), PET (positron emission tomography), MRI (magnetic resonance imaging), RT (radiotherapy), IMRT (intensity-modulated radiotherapy), Gy (Gray), AUC (area under the curve), ECOG (Eastern Cooperative Oncology Group), TNM (tumor, nodes, metastasis), BMI (body mass index), NOS (not otherwise specified), NLR (neutrophil-to-lymphocyte ratio), LMR (lymphocyte-to-monocyte ratio), ROC (receiver operating characteristics).

\section{References}

1. Sung, H.; Ferlay, J.; Siegel, R.L.; Laversanne, M.; Soerjomataram, I.; Jemal, A.; Bray, F. Global Cancer Statistics 2020: GLOBOCAN Estimates of Incidence and Mortality Worldwide for 36 Cancers in 185 Countries. CA Cancer J. Clin. 2021, 71, 209-249. [CrossRef]

2. National Cancer Institute. SEER*Explorer: An Interactive Website for SEER Cancer Statistics. Surveillance Research Program.

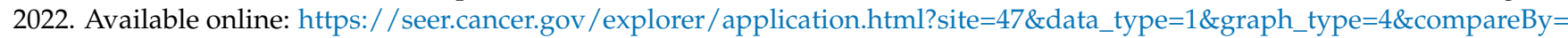
sex\&chk_sex_1=1\&chk_sex_3=3\&chk_sex_2=2\&race=1\&age_range=1\&advopt_precision=1\&advopt_display=2 (accessed on 10 January 2022).

3. Spigel, D.R.; Faivre-Finn, C.; Gray, J.E.; Vicente, D.; Planchard, D.; Paz-Ares, L.G.; Vansteenkiste, J.F.; Garassino, M.C.; Hui, R.; Quantin, X.; et al. Five-year survival outcomes with durvalumab after chemoradiotherapy in unresectable stage III NSCLC: An update from the PACIFIC trial. J. Clin. Oncol. 2021, 39, 8511. [CrossRef] 
4. Greten, F.R.; Grivennikov, S.I. Inflammation and Cancer: Triggers, Mechanisms, and Consequences. Immunity 2019, 51, 27-41. [CrossRef]

5. Lippitz, B.E.; Harris, R.A. Cytokine patterns in cancer patients: A review of the correlation between interleukin 6 and prognosis OncoImmunology 2016, 5, e1093722. [CrossRef]

6. Pikarsky, E.; Porat, R.M.; Stein, I.; Abramovitch, R.; Amit, S.; Kasem, S.; Gutkovich-Pyest, E.; Urieli-Shoval, S.; Galun, E.; Ben-Neriah, Y. NF-kB functions as a tumour promoter in inflammation-associated cancer. Nature 2004, 431, 461-466. [CrossRef]

7. Castell, J.V.; Gómez-Lechón, M.J.; David, M.; Hirano, T.; Kishimoto, T.; Heinrich, P.C. Recombinant human interleukin-6 (IL-6/BSF-2/HSF) regulates the synthesis of acute phase proteins in human hepatocytes. FEBS Lett. 1988, 232, 347-350. [CrossRef]

8. Sproston, N.R.; Ashworth, J.J. Role of C-Reactive Protein at Sites of Inflammation and Infection. Front. Immunol. 2018, 9, 754. [CrossRef]

9. Alsaffar, H.; Martino, N.; Garrett, J.P.; Adam, A.P. Interleukin-6 promotes a sustained loss of endothelial barrier function via Janus kinase-mediated STAT3 phosphorylation and de novo protein synthesis. Am. J. Physiol. Physiol. 2018, 314, C589-C602. [CrossRef]

10. Keller, U. Nutritional Laboratory Markers in Malnutrition. J. Clin. Med. 2019, 8, 775. [CrossRef]

11. Liao, W.S.; Jefferson, L.S.; Taylor, J.M. Changes in plasma albumin concentration, synthesis rate, and mRNA level during acute inflammation. Am. J. Physiol. Physiol. 1986, 251, C928-C934. [CrossRef]

12. Eckart, A.; Struja, T.; Kutz, A.; Baumgartner, A.; Baumgartner, T.; Zurfluh, S.; Neeser, O.; Huber, A.; Stanga, Z.; Mueller, B.; et al Relationship of Nutritional Status, Inflammation, and Serum Albumin Levels During Acute Illness: A Prospective Study. Am. J. Med. 2020, 133, 713-722. [CrossRef]

13. Sheinenzon, A.; Shehadeh, M.; Regina, M.; Shaoul, E.; Ronen, O. Serum albumin levels and inflammation. Int. J. Biol. Macromol. 2021, 184, 857-862. [CrossRef]

14. Kuboki, A.; Kanaya, H.; Nakayama, T.; Konno, W.; Goto, K.; Nakajima, I.; Kashiwagi, T.; Hirabayashi, H.; Haruna, S. Prognostic value of $\mathrm{C}$-reactive protein/albumin ratio for patients with hypopharyngeal and laryngeal cancer undergoing invasive surgery involving laryngectomy. Head Neck 2019, 41, 1342-1350. [CrossRef]

15. Shibutani, M.; Maeda, K.; Nagahara, H.; Iseki, Y.; Ikeya, T.; Hirakawa, K. Prognostic Significance of the Preoperative Ratio of C-Reactive Protein to Albumin in Patients with Colorectal Cancer. Anticancer Res. 2016, 36, 995-1001.

16. Martin, D.; Rödel, F.; Balermpas, P.; Winkelmann, R.; Fokas, E.; Rödel, C. C-Reactive Protein-to-Albumin Ratio as Prognostic Marker for Anal Squamous Cell Carcinoma Treated with Chemoradiotherapy. Front. Oncol. 2019, 9, 1200. [CrossRef]

17. Guo, Y.; Cai, K.; Mao, S.; Zhang, J.; Wang, L.; Zhang, Z.; Liu, M.; Zhang, W.; Wu, Y.; Yan, Y.; et al. Preoperative C-reactive protein/albumin ratio is a significant predictor of survival in bladder cancer patients after radical cystectomy: A retrospective study. Cancer Manag. Res. 2018, 10, 4789-4804. [CrossRef]

18. Araki, T.; Tateishi, K.; Sonehara, K.; Hirota, S.; Komatsu, M.; Yamamoto, M.; Kanda, S.; Kuraishi, H.; Hanaoka, M.; Koizumi, T. Clinical utility of the C-reactive protein:albumin ratio in non-small cell lung cancer patients treated with nivolumab. Thorac. Cancer 2021, 12, 603-612. [CrossRef]

19. Koh, Y.W.; Lee, H.W. Prognostic impact of C-reactive protein/albumin ratio on the overall survival of patients with advanced nonsmall cell lung cancers receiving palliative chemotherapy. Medicine 2017, 96, e6848. [CrossRef]

20. Zhang, F.; Ying, L.; Jin, J.; Chen, K.; Zhang, N.; Wu, J.; Zhang, Y.; Su, D. The C-reactive protein/albumin ratio predicts long-term outcomes of patients with operable non-small cell lung cancer. Oncotarget 2016, 8, 8835-8842. [CrossRef]

21. Yang, J.-R.; Xu, J.-Y.; Chen, G.-C.; Yu, N.; Yang, J.; Zeng, D.-X.; Gu, M.-J.; Li, D.-P.; Zhang, Y.-S.; Qin, L.-Q. Post-diagnostic C-reactive protein and albumin predict survival in Chinese patients with non-small cell lung cancer: A prospective cohort study. Sci. Rep. 2019, 9, 8143. [CrossRef]

22. Yotsukura, M.; Ohtsuka, T.; Kaseda, K.; Kamiyama, I.; Hayashi, Y.; Asamura, H. Value of the Glasgow Prognostic Score as a Prognostic Factor in Resectable Non-Small Cell Lung Cancer. J. Thorac. Oncol. 2016, 11, 1311-1318. [CrossRef]

23. Kishi, T.; Matsuo, Y.; Ueki, N.; Iizuka, Y.; Nakamura, A.; Sakanaka, K.; Mizowaki, T.; Hiraoka, M. Pretreatment Modified Glasgow Prognostic Score Predicts Clinical Outcomes After Stereotactic Body Radiation Therapy for Early-Stage Non-Small Cell Lung Cancer. Int. J. Radiat. Oncol. 2015, 92, 619-626. [CrossRef]

24. Leung, E.Y.; Scott, H.R.; McMillan, D.C. Clinical Utility of the Pretreatment Glasgow Prognostic Score in Patients with Advanced Inoperable Non-Small Cell Lung Cancer. J. Thorac. Oncol. 2012, 7, 655-662. [CrossRef]

25. Forrest, L.M.; McMillan, D.C.; McArdle, C.S.; Angerson, W.J.; Dunlop, D.J. Evaluation of cumulative prognostic scores based on the systemic inflammatory response in patients with inoperable non-small-cell lung cancer. Br. J. Cancer 2003, 89, 1028-1030. [CrossRef]

26. Peduzzi, P.; Concato, J.; Feinstein, A.R.; Holford, T.R. Importance of events per independent variable in proportional hazards regression analysis II. Accuracy and precision of regression estimates. J. Clin. Epidemiol. 1995, 48, 1503-1510. [CrossRef]

27. Grivennikov, S.I.; Greten, F.R.; Karin, M. Immunity, inflammation, and cancer. Cell 2010, 140, 883-899. [CrossRef]

28. Knittelfelder, O.; Delago, D.; Jakse, G.; Lukasiak, K.; Thurner, E.-M.; Thurnher, D.; Pichler, M.; Renner, W.; Stranzl-Lawatsch, H.; Langsenlehner, T. The Pre-Treatment C-Reactive Protein Represents a Prognostic Factor in Patients with Oral and Oropharyngeal Cancer Treated with Radiotherapy. Cancers 2020, 12, 626. [CrossRef]

29. Woo, H.D.; Kim, K.; Kim, J. Association between preoperative C-reactive protein level and colorectal cancer survival: A meta-analysis. Cancer Causes Control 2015, 26, 1661-1670. [CrossRef] 
30. Babic, A.; Schnure, N.; Neupane, N.P.; Zaman, M.M.; Rifai, N.; Welch, M.W.; Brais, L.K.; Rubinson, D.A.; Morales-Oyarvide, V.; Yuan, C.; et al. Plasma inflammatory cytokines and survival of pancreatic cancer patients. Clin. Transl. Gastroenterol. 2018, 9, e145. [CrossRef]

31. Hashimoto, K.; Ikeda, Y.; Korenaga, D.; Tanoue, K.; Hamatake, M.; Kawasaki, K.; Yamaoka, T.; Iwatani, Y.; Akazawa, K.; Takenaka, $\mathrm{K}$. The impact of preoperative serum C-reactive protein on the prognosis of patients with hepatocellular carcinoma. Cancer 2005, 103, 1856-1864. [CrossRef]

32. Kramer, M.; Heinisch, A.; Wegener, G.; Abbas, M.; Von Klot, C.; Peters, I.; Tezval, H.; Herrmann, T.; Kuczyk, M.; Merseburger, A C-reaktives Protein vor radikaler Zystektomie. Der. Urol. 2013, 53, 222-227. [CrossRef] [PubMed]

33. Yu, X.; Zhao, J.; Shi, L.; Jin, Y.; Sun, Y.; Shi, X. Prognostic value of circulating C-reactive protein levels in patients with non-small cell lung cancer: A systematic review with meta-analysis. J. Cancer Res. Ther. 2014, 10, 160-166. [CrossRef] [PubMed]

34. Mandaliya, H.; Jones, M.; Oldmeadow, C.; Nordman, I.I.C. Prognostic biomarkers in stage IV non-small cell lung cancer (NSCLC): Neutrophil to lymphocyte ratio (NLR), lymphocyte to monocyte ratio (LMR), platelet to lymphocyte ratio (PLR) and advanced lung cancer inflammation index (ALI). Transl. Lung Cancer Res. 2019, 8, 886-894. [CrossRef] [PubMed]

35. Russo, A.; Russano, M.; Franchina, T.; Migliorino, M.R.; Aprile, G.; Mansueto, G.; Berruti, A.; Falcone, A.; Aieta, M.; Gelibter, A.; et al. Neutrophil-to-Lymphocyte Ratio (NLR), Platelet-to-Lymphocyte Ratio (PLR), and Outcomes with Nivolumab in Pretreated Non-Small Cell Lung Cancer (NSCLC): A Large Retrospective Multicenter Study. Adv. Ther. 2020, 37, $1145-1155$. [CrossRef]

36. Palomar-Abril, V.; Soria-Comes, T.; Campos, S.T.; Ureste, M.M.; Bosch, V.G.; Maiques, I.C.M. Dynamic evaluation of neutrophilto-lymphocyte ratio as prognostic factor in stage III non-small cell lung cancer treated with chemoradiotherapy. Clin. Transl. Oncol. 2020, 22, 2333-2340. [CrossRef]

37. Kanzaki, H.; Hamamoto, Y.; Nagasaki, K.; Kozuki, T. Impact of neutrophil-to-lymphocyte ratio throughout the course of chemoradiotherapy on overall survival and distant failure in unresectable stage III non-small cell lung cancer. Jpn. J. Radiol. 2021, 39, 914-922. [CrossRef]

38. Coffelt, S.B.; Wellenstein, M.D.; De Visser, K.E. Neutrophils in cancer: Neutral no more. Nat. Rev. Cancer 2016, 16, 431-446. [CrossRef]

39. Li, W.; Ma, G.; Wu, Q.; Deng, Y.; Liu, Y.; Wang, J. Prognostic value of lymphocyte-to-monocyte ratio among Asian lung cancer patients: A systematic review and meta-analysis. Oncotarget 2017, 8, 110606-110613. [CrossRef]

40. Sekine, K.; Kanda, S.; Goto, Y.; Horinouchi, H.; Fujiwara, Y.; Yamamoto, N.; Motoi, N.; Ohe, Y. Change in the lymphocyte-tomonocyte ratio is an early surrogate marker of the efficacy of nivolumab monotherapy in advanced non-small-cell lung cancer. Lung Cancer 2018, 124, 179-188. [CrossRef]

41. Venkatesulu, B.P.; Mallick, S.; Lin, S.H.; Krishnan, S. A systematic review of the influence of radiation-induced lymphopenia on survival outcomes in solid tumors. Crit. Rev. Oncol. 2018, 123, 42-51. [CrossRef]

42. Dhanapal, R.; Saraswathi, T.; Govind, R.N. Cancer cachexia. J. Oral Maxillofac. Pathol. 2011, 15, 257-260. [CrossRef]

43. Almasaudi, A.S.; Dolan, R.D.; Edwards, C.A.; McMillan, D.C. Hypoalbuminemia Reflects Nutritional Risk, Body Composition and Systemic Inflammation and is Independently Associated with Survival in Patients with Colorectal Cancer. Cancers 1986, 12, 1986. [CrossRef] [PubMed]

44. Lim, W.S.; Roh, J.-L.; Kim, S.-B.; Choi, S.-H.; Nam, S.Y.; Kim, S.Y. Pretreatment albumin level predicts survival in head and neck squamous cell carcinoma. Laryngoscope 2017, 127, E437-E442. [CrossRef]

45. Tanriverdi, O.; Avci, N.; Oktay, E.; Kalemci, S.; Pilanci, K.N.; Cokmert, S.; Menekse, S.; Kocar, M.; Sen, C.A.; Akman, T.; et al Pretreatment Serum Albumin Level is an Independent Prognostic Factor in Patients with Stage IIIB Non-Small Cell Lung Cancer: A Study of the Turkish Descriptive Oncological Researches Group. Asian Pac. J. Cancer Prev. 2015, 16, 5971-5976. [CrossRef] [PubMed]

46. Stares, M.; Swan, A.; Cumming, K.; Ding, T.-E.; Leach, J.; Stratton, C.; Thomson, F.; Barrie, C.; MacLennan, K.; Campbell, S.; et al Hypoalbuminaemia as a Prognostic Biomarker of First-Line Treatment Resistance in Metastatic Non-small Cell Lung Cancer. Front. Nutr. 2021, 8, 734735. [CrossRef] [PubMed]

47. Rim, C.H.; Yoon, W.S.; Lee, J.A.; Yang, D.S.; Lee, N.K.; Park, Y.J.; Kim, C.Y. Factors predicting intolerance to definitive conventional radiotherapy in geriatric patients. Strahlenther. Onkol. 2018, 194, 894-903. [CrossRef]

48. Yu, J.M.; Yang, M.; Xu, H.X.; Li, W.; Fu, Z.; Lin, Y.; Shi, Y.Y.; Song, C.H.; Shi, H.P.; Guo, Z.Q.; et al. Association Between Serum C-Reactive Protein Concentration and Nutritional Status of Malignant Tumor Patients. Nutr. Cancer 2018, 71, 240-245. [CrossRef]

49. Zhu, L.; Chen, S.; Ma, S.; Zhang, S. Glasgow prognostic score predicts prognosis of non-small cell lung cancer: A meta-analysis. SpringerPlus 2016, 5, 439. [CrossRef]

50. Jin, J.; Hu, K.; Zhou, Y.; Li, W. Clinical utility of the modified Glasgow prognostic score in lung cancer: A meta-analysis. PLoS ONE 2017, 12, e0184412. [CrossRef]

51. Matsumoto, T.; Itoh, S.; Yoshizumi, T.; Kurihara, T.; Yoshiya, S.; Mano, Y.; Takeishi, K.; Harada, N.; Ikegami, T.; Soejima, Y.; et al. C-reactive protein: Albumin ratio in patients with resectable intrahepatic cholangiocarcinoma. BJS Open 2020, 4, 1146-1152. [CrossRef]

52. Icard, P.; Schussler, O.; Loi, M.; Bobbio, A.; Lupo, A.M.; Wislez, M.; Iannelli, A.; Fournel, L.; Damotte, D.; Alifano, M. Pre-Disease and Pre-Surgery BMI, Weight Loss and Sarcopenia Impact Survival of Resected Lung Cancer Independently of Tumor Stage. Cancers 2020, 12, 266. [CrossRef] [PubMed] 
53. Fukui, T.; Taniguchi, T.; Kawaguchi, K.; Fukumoto, K.; Nakamura, S.; Sakao, Y.; Yokoi, K. Comparisons of the clinicopathological features and survival outcomes between lung cancer patients with adenocarcinoma and squamous cell carcinoma. Gen. Thorac. Cardiovasc. Surg. 2015, 63, 507-513. [CrossRef] [PubMed]

54. Wheatley-Price, P.; Blackhall, F.; Lee, S.M.; Ma, C.; Ashcroft, L.; Jitlal, M.; Qian, W.; Hackshaw, A.; Rudd, R.; Booton, R.; et al. The influence of sex and histology on outcomes in non-small-cell lung cancer: A pooled analysis of five randomized trials. Ann. Oncol. 2010, 21, 2023-2028. [CrossRef] [PubMed]

55. Kale, M.S.; Mhango, G.; Gomez, J.; Sigel, K.; Smith, C.B.; Bonomi, M.; Wisnivesky, J.P. Treatment Toxicity in Elderly Patients with Advanced Non-Small Cell Lung Cancer. Am. J. Clin. Oncol. 2017, 40, 470-476. [CrossRef] [PubMed] 